United Nations Educational Scientific and Cultural Organization

and

International Atomic Energy Agency

THE ABDUS SALAM INTERNATIONAL CENTRE FOR THEORETICAL PHYSICS

\title{
TAME KERNELS FOR BIQUADRATIC NUMBER FIELDS
}

\author{
Qin Yue ${ }^{1}$ \\ Mathematics Department, Nanjing University of Aeronautics and Astronautics, \\ Nanjing Jiangsu 210016, People's Republic of China \\ and
}

The Abdus Salam International Centre for Theoretical Physics, Trieste, Italy.

\begin{abstract}
Let $F=\mathbb{Q}\left(\sqrt{-d_{1}}\right)$ and $E=\mathbb{Q}\left(\sqrt{-d_{1}}, \sqrt{d_{2}}\right), d_{1}$ and $d_{2}$ squarefree integers, be an imaginary field and a biquadratic field, respectively. Let $S$ be the set consisting of all infinite primes, all dyadic primes and all finite primes which ramify in $E$. Suppose the 4-rank of the class group of $F$ is zero and the $S$-ideal class group of $F$ has odd order, we give the forms of all elements of order $\leq 2$ in $\mathrm{K}_{2} \mathrm{O}_{E}$ and use the Hurrelbrink and Kolster's method [5] to obtain the forms of all elements of order 4 in $\mathrm{K}_{2} \mathrm{O}_{E}$.
\end{abstract}

MIRAMARE - TRIESTE

August 2004

\footnotetext{
${ }^{1}$ yueqin2@263.net
} 


\section{Introduction}

Let $E=F(\sqrt{d})$ be a relative quadratic extension over a number field $F$, and let $O_{E}$ and $O_{F}$ be the rings of integers of $E$ and $F$, respectively. Let $S$ be the finite set of primes of $F$ consisting of all dyadic primes, all finite primes that ramify in $E$ and all infinite primes and let $S_{f}$ be the set of finite primes in $S$. We will use the following notations for any abelian group $A:{ }_{2} A$ denotes the subgroup of elements of order $\leq 2$ of $A ; A(2)$ denotes the 2-Sylow subgroup of $A ; r_{2^{n}}(A)$ denotes the $2^{n}$-rank of $A$.

For an arbitrary number field $L$ with the ring of integers $O_{L}$, there is a surjective homomorphism

$$
K_{2} O_{L} \rightarrow \bigoplus_{v \text { real }} \mu_{2} \rightarrow 0
$$

induced by the Hilbert symbols at the real infinite primes. Hence its kernel denoted by $K_{2} O_{L}^{\prime}$ is of index $2^{r_{1}(L)}$ in $K_{2} O_{L}$.

First we recall some results in [5]. Hurrelbrink and Kolster determined the structure of the 2-Sylow subgroups $K_{2} O_{E}^{\prime}(2)$ and $K_{2} O_{E}(2)$, provided that $K_{2} O_{F}^{\prime}(2)=0$. If $K_{2} O_{F}^{\prime}(2)=0$, then $F$ has only one dyadic prime and the $S_{2}$-class group $C^{S_{2}}(F)$ has odd order, where $S_{2}$ is the set of all infinite primes and the dyadic prime. Let $\operatorname{Tr}(E / F)$ denote the kernel of the transfer map $($ see $[7])$

$$
\operatorname{Tr}_{E / F}: K_{2} E \rightarrow K_{2} F
$$

In [5], there is a homomorphism ${ }_{2} \phi_{E / F}:{ }_{2} \operatorname{Tr}(E / F) \rightarrow{ }_{2} \operatorname{Br}(F) /\left(-d, \Delta_{F}\right)$, where $\left(-d, \Delta_{F}\right)$ denotes the subgroup of the Brauer group of $F$ generated by all classes $(-d, \delta)$ with $\delta$ in the Tate kernel $\Delta_{F}$, satisfying $\{-1, \delta\}=1$ in $K_{2} F$; see [11].

Suppose ${ }_{2} \phi_{F(i) / F}$ is trivial and the $S$-class group $C^{S}(F)$ has odd order, then there exists an exact sequence (see [5, Corollary 3.9])

$$
0 \rightarrow\left({ }_{2} K_{2} O_{E}\right)^{2} \cap \operatorname{Tr}(E / F) \rightarrow{ }_{2} K_{2} O_{E} \cap \operatorname{Tr}(E / F) \stackrel{\psi_{E / F}}{\rightarrow} B r^{S_{f}}\left(E_{0} / F\right) /\left(-d, \Delta_{F}\right),
$$

where $B^{S_{f}}\left(E_{0} / F\right)$ consists of all classes of quaternion algebras of the form $(-d, a)$ with $a \in F^{*}$ which are locally trivial at primes in $S_{f}$. Hence we can use the above exact sequence to compute the 4-rank of $K_{2} O_{E} \cap \operatorname{Tr}(E / F)$. In fact, if $F$ is totally real or $K_{2} O_{F}^{\prime}(2)=0$, then ${ }_{2} \phi_{F(i) / F}$ is trivial.

In the paper, we will always assume that $F=\mathbb{Q}\left(\sqrt{-d_{1}}\right), d_{1}>2$ a squarefree integer, is an imaginary quadratic field and $E=F\left(\sqrt{d_{2}}\right), d_{2}$ a squarefree integer, is a biquadratic field. Let $C(F)$ and $C^{S}(F)$ denote the class group of $F$ and the $S$-ideal class group of $F$, respectively. We shall determine the structure of the 2-Sylow subgroup $\mathrm{K}_{2} \mathrm{O}_{E}(2)$ clearly, provided that $r_{4}(C(F))=0$ and $C^{S}(F)$ has odd order.

In section 2, we give the forms of all elements of order at most 2 in $K_{2} O_{E} \cap \operatorname{Tr}(E / F)$ via solutions of systems of $\mathbb{F}_{2}$-linear equations whose coefficient matrices consist of the Legendre 
symbols (Theorem 2.1); further, we obtain the forms of all elements of order at most 2 in $\mathrm{K}_{2} \mathrm{O}_{E}$ and calculate $r_{2}\left(K_{2} O_{E}\right)$ via the determination of the $\mathbb{F}_{2}$-ranks of certain matrices of Legendre symbols (Theorem 2.2).

In section 3, we mainly use the results of section 2 and [5] to investigate $r_{4}\left(K_{2} O_{E}\right)$. We supply Proposition 3.1 and Proposition 3.2 such that we can use the preceding exact sequence even if $F$ is an imaginary quadratic field with $r_{4}(C(F))=0$, which removes the restriction on a base field $F$ in [5]. Hence we use it to compute $r_{4}\left(K_{2} O_{E}\right)$ (Theorem 3.2); we also give an algorithm to get elements of order 2 and 4 of $K_{2} O_{E}$ via the determination of the $\mathbb{F}_{2}$-ranks of certain matrices of local Hilbert symbols analogous to the Rédei's matrix (Corollary 3.1). We also illustrate the method through various examples.

In this paper, we express the forms of all elements of order at most 4 in $K_{2} O_{E}$ by various $\mathbb{F}_{2^{-}}$ matrices consisting of Legendre symbols and local Hilbert symbols analogous to Rédei matrix, but it has to be pointed out that this paper uses the Hurrelbrink and Kolster's method of relative quadratic extension [5] to investigate specially biquadratic number fields $E$ and generalizes the results of Browkin, Schinzel [1] and Qin [9,10].

In this paper, we always assume that $F=\mathbb{Q}\left(\sqrt{-d_{1}}\right)$ and $E=\mathbb{Q}\left(\sqrt{-d_{1}}, \sqrt{d_{2}}\right), d_{1}>2$ and $d_{2}$ squarefree integers, are an imaginary quadratic field and a biquadratic field, respectively.

\section{2-rank}

Let $S$ be the finite set of primes in $F$ consisting of all infinite primes, all dyadic primes, all finite primes ramifying in $E$. In this section, we will give elements of order $\leq 2$ of $K_{2} O_{E}$, provided the 4-rank of the class group $C(F)$ is 0 and the $S$-ideal class group $C^{S}(F)$ has odd order.

Suppose $r_{4}(C(F))=0$, we observe the 2-Sylow subgroup of $K_{2} O_{F}$ and the Tate kernel $\Delta_{F}$.

Proposition 2.1 Let $F=\mathbb{Q}\left(\sqrt{-d_{1}}\right), d_{1}>2$ a squarefree integer, be an imaginary quadratic number field and $r_{4}(C(F))=0$.

(1) If $d_{1} \not \equiv \pm 1 \bmod 8$ or $d_{1} \equiv-1 \bmod 8$ with $2 \notin N F$, then $r_{4}\left(K_{2} O_{F}\right)=0$ and $\Delta_{F}=$ $\{2, m\} F^{* 2}, m \mid d_{1}$.

(2) If $d_{1} \equiv 1 \bmod 8$, then $r_{4}\left(K_{2} O_{F}\right)=1, r_{8}\left(K_{2} O_{F}\right)=0$ and $\Delta_{F}=\{2, m\} F^{* 2}, m \mid d_{1}$.

(3) If $d_{1} \equiv-1 \bmod 8$ with $2 \in N F$, then $r_{4}\left(K_{2} O_{F}\right)=0$ and $\Delta_{F}=\left\{2, m\left(u+\sqrt{-d_{1}}\right)\right\} F^{* 2}$, where $m \mid d_{1}$ and $u^{2}-2 w^{2}=-d_{1}, u, w \in \mathbb{N}$.

Proof. Let $F_{0}=\mathbb{Q}\left(\sqrt{d}_{1}\right)$ be a real quadratic field versus $F=\mathbb{Q}\left(\sqrt{-d_{1}}\right)$ and $C\left(F_{0}\right)$ the narrow class group of $F_{0}$. We have the fact: $r_{4}\left(C\left(F_{0}\right)\right) \leq r_{4}(C(F)) \leq r_{4}\left(C\left(F_{0}\right)\right)+1$; compare e.g. [12]. Since $r_{4}(C(F))=0, r_{4}\left(C\left(F_{0}\right)\right)=0$, so $2 \notin N F$ if $d_{1} \not \equiv-1 \bmod 8$.

(1) If $d_{1} \not \equiv \pm 1 \bmod 8$ or $d_{1} \equiv-1 \bmod 8$ with $2 \notin N F$, then, by [17, theorem 4.1], $r_{4}\left(K_{2} O_{F}\right)=$ 0 , so $\Delta_{F}=\{2, m\} F^{* 2}, m \mid d_{1}$, by [1] and [11].

(2) If $d_{1} \equiv 1 \bmod 8$, then $r_{4}(C(F))=r_{4}\left(C\left(F_{0}\right)\right)=0$ and $2 \notin N F$. By [12, theorem 1(i)], there is a positive divisor $n \equiv \pm 3 \bmod 8$ of $d_{1}$ such that the equation $n z^{2}=x^{2}-d_{1} y^{2}$ is solvable 
over $\mathbb{Z}$. By [14, theorem 4.1], $r_{4}\left(K_{2} O_{F}\right)=1, r_{8}\left(K_{2} O_{F}\right)=0$ and $1 \neq\{-1, n\} \in\left(K_{2} O_{F}\right)^{2}$. By [1] and $2 \notin N F$, we get $\Delta_{F}=\{2, m\} F^{* 2}, m \mid d_{1}$.

(3) If $d_{1} \equiv-1 \bmod 8$ with $2 \in N F$, then $r_{4}(C(F))=r_{4}\left(C\left(F_{0}\right)\right)=0$, so the class $[\mathcal{D}]=1$ in $C\left(F_{0}\right)$, where $\mathcal{D}$ is a dyadic ideal of $F_{0}$. By [17, theorem 4.1 and 4.3] or [16], $r_{4}\left(K_{2} O_{F}\right)=0$ and $m\left(u+\sqrt{-d_{1}}\right) \in \Delta_{F}, m \mid d_{1},-d_{1}=u^{2}-2 w^{2}, u, w \in \mathbb{N}$.

Let $S$ be a finite set of primes of $F$ including all infinite primes and all dyadic primes. We will give a method to determine that the $S$-ideal class group $C^{S}(F)$ has odd order, provided $r_{4}(C(F))=0$. For convenience, we make a definition, which will always be applied for in this paper.

Definition 2.1 Let $F=\mathbb{Q}\left(\sqrt{-d_{1}}\right)$ be an imaginary quadratic field. Set

$$
S^{\prime}=\left\{q_{0}=2, q_{1}, \cdots, q_{s}, q_{s+1} \cdots, q_{s+r}\right\}
$$

where $S^{\prime}$ is the set of primes of $\mathbb{Q}$, odd primes $q_{1}, \cdots, q_{s}$ are decomposable in $F$ and odd primes $q_{s+1} \cdots, q_{s+r}$ are inert in $F$. We define

$$
\begin{gathered}
S=\left\{\text { all primes } Q \text { of } F|Q| \infty \text { or } Q \mid q_{i} \text { for some } q_{i} \text { in } S^{\prime}\right\} \\
S_{f}=\left\{\text { all primes } Q \text { of } F|Q| q_{i} \text { for some } q_{i} \text { in } S^{\prime}\right\} .
\end{gathered}
$$

In fact, $\left|S_{f}\right|=2 s+r+s_{2}$, where $s_{2}$ is the number of dyadic primes of $F$.

In this paper, we will always assume that $p_{1}, \cdots, p_{t}$ are all primes which ramify in $F$ and $p_{t}=2$ if $2 \mid D_{F}$, where $D_{F}$ is the discriminant of $F$. Let $M_{F}=\left(a_{i j}\right)$ be the $t \times t$ Rédei's matrix of $F$ with coefficients $a_{i j} \in \mathbb{F}_{2}$, where

$$
(-1)^{a_{i j}}=\left\{\begin{array}{ll}
\left(\frac{p_{j}}{p_{i}}\right) & \text { if } i \neq j, \\
\left(\frac{D_{F} / p_{i}^{*}}{p_{i}}\right) & \text { if } i=j,
\end{array} \quad a_{i j}= \begin{cases}\left(\frac{p_{j}}{p_{i}}\right)^{\prime} & \text { if } i \neq j, \\
\left(\frac{D_{F} / p_{i}^{*}}{p_{i}}\right)^{\prime} & \text { if } i=j,\end{cases}\right.
$$

$p_{i}^{*}$ the discriminant of $p_{i}$. Let $M_{F}^{\prime}$ be the $(t-1) \times t$ matrix by deleting the $t$-th row of $M_{F}$. It is clear that $r_{4}(C(F))=0$ if and only if $\operatorname{rank} M_{F}=\operatorname{rank} M_{F}^{\prime}=t-1$.

Set the matrix over $\mathbb{F}_{2}$

$$
M^{S}=\left(\begin{array}{llll}
\left(\frac{2}{p_{1}}\right)^{\prime} & \left(\frac{q_{1}}{p_{1}}\right)^{\prime} & \cdots & \left(\frac{q_{s}}{p_{1}}\right)^{\prime} \\
\vdots & \vdots & & \vdots \\
\left(\frac{2}{p_{t-1}}\right)^{\prime} & \left(\frac{q_{1}}{p_{t-1}}\right)^{\prime} & \cdots & \left(\frac{q_{s}}{p_{t-1}}\right)^{\prime}
\end{array}\right) \text { or }\left(\begin{array}{ccc}
\left(\frac{q_{1}}{p_{1}}\right)^{\prime} & \cdots & \left(\frac{q_{s}}{p_{1}}\right)^{\prime} \\
\vdots & & \vdots \\
\left(\frac{q_{1}}{p_{t-1}}\right)^{\prime} & \cdots & \left(\frac{q_{s}}{p_{t-1}}\right)^{\prime}
\end{array}\right) \text {, }
$$

where $M^{S}$ is the $(t-1) \times s$ matrix only if $-d_{1} \equiv 5 \bmod 8$ and $M^{S}$ is the $(t-1) \times(s+1)$ matrix otherwise.

Proposition 2.2 If $r_{4}(C(F))=0$, then the $S$-ideal class group $C^{S}(F)$ has odd order if and only if $\operatorname{rank}^{S}=t-1$. 
Proof. Without loss of generality, suppose $-d_{1} \not \equiv 5 \bmod 8$. Take $Q_{0}=\mathcal{D}$ a dyadic ideal and $Q_{j}$ integral ideals of $F$ over $q_{j}, j=1, \cdots, s+r$, respectively. Let $G=\left(\left\{\left[Q_{j}\right] \mid j=0,1, \cdots, s\right\}\right)$ be the subgroup of $C(F)$ generated by the ideal classes $\left[Q_{j}\right], j=0,1, \cdots, s$ (in fact: $\left[Q_{j}\right]=1, j>s$, by $q_{j}$ inert in $\left.F\right)$, then $C^{S}(F)=C(F) / G$.

If $\operatorname{rank} M^{S}=t-1$, then, without loss of generality, we assume that the beginning $t-1$ columns of $M^{S}$ are linear independent. Hence each column of $M_{F}^{\prime}$ can be linearly expressed by the beginning $t-1$ columns of $M^{S}$ over $\mathbb{F}_{2}$ :

$$
\left(\begin{array}{c}
\left(\frac{p_{i}}{p_{1}}\right)^{\prime} \\
\vdots \\
\left(\frac{p_{i}}{p_{t-1}}\right)^{\prime}
\end{array}\right)=\left(\begin{array}{c}
\left(\frac{q_{i_{1}} \cdots q_{i_{j}}}{p_{1}}\right)^{\prime} \\
\vdots \\
\left(\frac{q_{i_{1}} \cdots q_{i_{j}}}{p_{t-1}}\right)^{\prime}
\end{array}\right) .
$$

Therefore, by Legendre theorem (see [17]), the Diophantine equation $p_{i} q_{i_{1}} \cdots q_{i_{j}} z^{2}=x^{2}+d_{1} y^{2}$ has a nontrivial and relatively prime solution $(x, y, z)=(a, b, c)$ over $\mathbb{Z}$. Hence, in $C(F)$, $\left[P_{i}\right]\left[Q_{i_{1}} \cdots Q_{i_{j}}\right]=\left[P_{c}\right]^{2}$, where $P_{i}$ is the ambiguous ideal over $p_{i}$ and $P_{c}$ is a ideal over $c$. Since $r_{4}(C(F))=0$, there is an odd number $k$ such that $\left[P_{i}\right]=\left[Q_{i_{1}} \cdots Q_{i_{j}}\right]^{k} \in G$. Hence each ambiguous ideal class $\left[P_{i}\right]$ belongs to $G$ by the genus theory (see [4]).

Suppose that an ideal class $[X]$ of $F$ satisfies $[X]^{2} \equiv 1 \bmod G$. Since $r_{4}(C(F))=0$, there is an ambiguous ideal class $[P]$ such that $[P][X]$ has odd order in the class group $C(F)$. By $([P][X])^{2}=[X]^{2} \in G,[P][X] \in G$, so $[X] \in G$. Hence $C^{S}(F)$ has odd order.

Conversely, if $C^{S}(F)$ has odd order, then each ambiguous ideal class $\left[P_{i}\right]$ over $p_{i}$ belongs to $G$. Hence $\left[P_{i}\right]\left[Q_{i_{1}} \cdots Q_{i_{j}}\right] \in G^{2} \subset C(F)^{2}$, so the Diophantine equation $p_{i} q_{i_{1}} \cdots q_{i_{j}} z^{2}=x^{2}+d_{1} y^{2}$ has nontrivial solutions over $\mathbb{Z}$. By Legendre theorem (see [17]), the i-th column of $M_{F}^{\prime}$ is linearly expressed by columns of $M^{S}$ over $\mathbb{F}_{2}$. Hence each column of $M_{F}^{\prime}$ can be linearly expressed by columns of $M^{S}$ over $\mathbb{F}_{2}$. So $\operatorname{rank} M^{S}=t-1$ by $\operatorname{rank} M_{F}^{\prime}=t-1$.

Let $E=\mathbb{Q}\left(\sqrt{-d_{1}}, \sqrt{d_{2}}\right)$ be a biquadratic field. In this paper, we will assume that $S$ defined as Definition 2.1 is the set of all infinite primes, all dyadic primes, and all finite primes which ramify in $E$. In fact, $d_{2}=n q_{1} \cdots q_{s+r}, n \mid 2 d_{1}$, is a squarefree integer. Suppose $r_{4}(C(F))=0$ and $C^{S}(F)$ has odd order, we will give all elements of order 2 of $\mathrm{K}_{2} \mathrm{O}_{E}$.

For an arbitrary number field $L$ with the ring of integers $O_{L}$ we define

$$
D_{L}=\left\{x \in L^{*} \mid v_{P}(x) \equiv 0 \bmod 2 \text { for all finite primes } P \nmid 2\right\} \text {. }
$$

We obtain an exact sequence

$$
0 \rightarrow \widetilde{\Delta}_{L} \rightarrow D_{L} / L^{* 2} \rightarrow{ }_{2} K_{2} O_{L} \rightarrow 0
$$

where $\Delta_{L}$ is the Tate kernel of $L$ and $\widetilde{\Delta}_{L}=\Delta_{L} / L^{* 2}$. Let

$$
R(E / F)=\left\{x \in E^{*} \mid N_{E / F}(x) \in \Delta_{F}\right\}, D(E / F)=R(E / F) \cap D_{E} .
$$


We define $\epsilon$ via

$$
2^{\epsilon}=\left[\Delta_{F}: \Delta_{F} \cap N_{E / F}\left(E^{*}\right)\right] .
$$

Let $\operatorname{Tr}(E / F)$ denote the kernel of the transfer map $\operatorname{Tr}_{E / F}: K_{2} E \rightarrow K_{2} F$. It is clear that there is an exact sequence

$$
0 \rightarrow \widetilde{\Delta}_{E} \rightarrow D(E / F) / E^{* 2} \rightarrow{ }_{2} K_{2} O_{E} \cap \operatorname{Tr}(E / F) \rightarrow 0 .
$$

Suppose $C^{S}(F)$ has odd order, by [5, lemma 2.4 and proposition 2.6], there is an exact sequence

$$
0 \rightarrow \mathbb{Z} / 2 \mathbb{Z} \rightarrow U^{S} /\left(U^{S}\right)^{2} \rightarrow D(E / F) / E^{* 2} \rightarrow\left(\Delta_{F} \cap N_{E / F}\left(E^{*}\right)\right) / F^{* 2} \rightarrow 0,
$$

where $U^{S}$ is the group of units of the ring of $S$-integers $O_{F}^{S}$. Hence

$$
r_{2}\left(D(E / F) / E^{* 2}\right)=r_{1}+r_{2}+\left|S_{f}\right|+r_{2}+1-\epsilon-1=\left|S_{f}\right|+2-\epsilon,
$$

where $S_{f}$ consists of all finite primes in $S, \epsilon$ is defined as (2.3), $r_{1}=0$ and $r_{2}=1\left(r_{1}\right.$ and $r_{2}$ are the number of real and pairs of complex primes of $F$, respectively). By $(2.4)$ and $r_{2}\left(\widetilde{\Delta}_{E}\right)=3$, we get the result of [5, remark 2.8]:

$$
r_{2}\left(K_{2} O_{E} \cap \operatorname{Tr}(E / F)\right)=\left|S_{f}\right|-1-\epsilon=2 s+r+s_{2}-1-\epsilon .
$$

Suppose $r_{4}(C(F))=0$ and $C^{S}(F)$ has odd order, then $\operatorname{rank} M^{S}=t-1$ by Proposition 2.2 and $2 \notin N F$ if $-d_{2} \not \equiv 1 \bmod 8$. In the following, we give the representative set of $D(E / F) / E^{* 2}$.

Theorem 2.1 (1) If $2 \mid D_{F}$ and the rank of the following matrix is $t-1$ :

$$
M_{1}^{S}=\left(\begin{array}{cccc}
\left(\frac{2}{p_{1}}\right)^{\prime} & \left(\frac{q_{1}}{p_{1}}\right)^{\prime} & \cdots & \left(\frac{q_{t-2}}{p_{1}}\right)^{\prime} \\
\vdots & \vdots & & \vdots \\
\left(\frac{2}{p_{t-1}}\right)^{\prime} & \left(\frac{q_{1}}{p_{t-1}}\right)^{\prime} & \cdots & \left(\frac{q_{t-2}}{p_{t-1}}\right)^{\prime}
\end{array}\right)
$$

then the representative set of $D(E / F) / E^{* 2}$ is

$$
\left\{p_{1}, \cdots, p_{t}=2, q_{2}, \cdots, q_{s+r}, a_{t-1}+\sqrt{-d_{1}} b_{t-1}, \cdots, a_{s}+\sqrt{-d_{1}} b_{s}\right\} \cup T
$$

where, for each $q_{i}(i=t-1, \cdots, s)$, there is $\left\{q_{i_{1}}, \cdots, q_{i_{j}}\right\} \subset\left\{2, q_{1}, \cdots, q_{t-2}\right\}$ such that $q_{i} q_{i_{1}} \cdots q_{i_{j}} z^{2}=$ $x^{2}+d_{1} y^{2}$ has a relatively prime solution $(x, y, z)=\left(a_{j}, b_{j}, c_{j}\right)$ over $\mathbb{Z}$, and

$$
T= \begin{cases}\left\{e_{1}+\sqrt{d_{2}} f_{1}, e_{2}+\sqrt{d_{2}} f_{2}\right\} & \text { if } \epsilon=0, \\ \left\{e_{1}+\sqrt{d_{2}} f_{1}\right\} & \text { if } \epsilon=1, \\ \emptyset & \text { if } \epsilon=2,\end{cases}
$$

with $e_{j}^{2}-d_{2} f_{j}^{2}=\delta_{j} \in \Delta_{F}, e_{j}, f_{j} \in F^{*}$.

(2) If $d_{1} \equiv-1 \bmod 8,2 \notin N F$ and the rank of the form (2.7) is $t-1$, then the representative set of $D(E / F) / E^{* 2}$ is

$$
\left\{p_{1}, \cdots, p_{t}, 2, q_{2}, \cdots, q_{s+r}, a_{t-1}+\sqrt{-d_{1}} b_{t-1}, \cdots, a_{s}+\sqrt{-d_{1}} b_{s}\right\} \cup T .
$$


(3) If $d_{1} \equiv-5 \bmod 8$ and the rank of the following matrix is $t-1$ :

$$
M_{2}^{S}=\left(\begin{array}{ccc}
\left(\frac{q_{1}}{p_{1}}\right)^{\prime} & \cdots & \left(\frac{q_{t-1}}{p_{1}}\right)^{\prime} \\
\vdots & & \vdots \\
\left(\frac{q_{1}}{p_{t-1}}\right)^{\prime} & \cdots & \left(\frac{q_{t-1}}{p_{t-1}}\right)^{\prime}
\end{array}\right),
$$

then the representative set of $D(E / F) / E^{* 2}$ is

$$
\left\{p_{1}, \cdots, p_{t}, 2, q_{2}, \cdots, q_{s+r}, a_{t}+\sqrt{-d_{1}} b_{t}, \cdots, a_{s}+\sqrt{-d_{1}} b_{s}\right\} \cup T
$$

(4) If $d_{1} \equiv-1 \bmod 8,2 \in N F$, and the rank of the form (2.8) is $t-1$, then the representative set of $D(E / F) / E^{* 2}$ is

$$
\left\{p_{1}, \cdots, p_{t}, 2, q_{2}, \cdots, q_{s+r}, u+\sqrt{-d_{1}}, a_{t}+\sqrt{-d_{1}} b_{t}, \cdots, a_{s}+\sqrt{-d_{1}} b_{s}\right\} \cup T,
$$

where $-d_{1}=u^{2}-2 w^{2}, u, w \in \mathbb{N}$.

Note: in (2), (3) and (4), $a_{i}+\sqrt{-d_{1}} b_{i}$ and $T$ are analogue to them in (1).

Proof. By the preceding discussion and the assumption, we get

$$
r_{2}\left(D(E / F) / E^{* 2}\right)=\left|S_{f}\right|+2-\epsilon=2 s+r+s_{2}+2-\epsilon,
$$

where $s_{2}$ is the number of Dyadic ideals in $F$.

(1) If $2 \mid D_{F}$, then $s_{2}=1$. By $\operatorname{rank} M_{1}^{S}=t-1$, each column $\left(\left(\frac{q_{i}}{p_{1}}\right)^{\prime}, \cdots,\left(\frac{q_{i}}{p_{t-1}}\right)^{\prime}\right)^{T}$ of $M^{S}$ can be expressed by the columns of $M_{1}^{S}$, i.e.,

$$
\left(\begin{array}{c}
\left(\frac{q_{i} q_{i_{1}} \cdots q_{i_{j}}}{p_{1}}\right)^{\prime} \\
\vdots \\
\left(\frac{q_{i} q_{i_{1} \cdots q_{i_{j}}}}{p_{t-1}}\right)^{\prime}
\end{array}\right)=0
$$

where $\left\{q_{i_{1}}, \cdots, q_{i_{j}}\right\} \subset\left\{2, q_{1}, \cdots, q_{t-1}\right\}$. By Legendre theorem (see [17]), the Diophantine equation $q_{i} q_{i_{1}} \cdots q_{i_{j}} z^{2}=x^{2}+d_{1} y^{2}$ has a relatively prime solution $(x, y, z)=\left(a_{i}, b_{i}, c_{i}\right)$ over $\mathbb{Z}$. Hence we get $\left\{p_{1}, \cdots, p_{t}, q_{2}, \cdots, q_{s+r}, a_{t-1}+\sqrt{-d_{1}} b_{t-1}, \cdots, a_{s}+\sqrt{-d_{1}} b_{s}\right\} \subseteq D(E / F)$ and such $2 s+r+1$ elements must be distinct modulo $E^{* 2}$ each other.

On the other hand, suppose $\delta \in \Delta_{F}, \delta \notin F^{* 2}$, is a norm from $E^{*}$, i.e., $\delta=N_{E / F}(\alpha)$, $\alpha \in E$, then clearly the valuation of $\alpha$ is even at all undecomposed non-dyadic finite primes and $v_{\mathcal{P}}(\alpha) \equiv v_{\mathcal{P}^{\sigma}}(\alpha) \bmod 2$ at finite decomposed primes $P=\mathcal{P}^{\sigma}$ of $F$. Since $C^{S}(F)$ has odd order, we can find $f \in F^{*}$ such that $\alpha \cdot f \in D(E / F)$, so $\alpha \cdot f \in T$.

By the property of norm, it is verified that each product of such distinct elements does not belong to $E^{* 2}$. Note the definition of $\epsilon$ in (2.3), then (1) holds.

In cases (2) and (4), $2 \nmid D$ and $s_{2}=2$; in case (3), $2 \nmid D$ and $s_{2}=1$. Similarly to case (1), we can prove them. 
Remark 2.1 (1) By Theorem 2.1 and (2.4), we have given all elements of order at most 2 in $K_{2} O_{E} \cap \operatorname{Tr}(E / F)$.

(2) Since $r_{4}(C(F))=0$, by Proposition 2.1 we get $\Delta_{F}=\{2, \delta\} F^{* 2}$, so $2, \delta \in \Delta_{E}$. Suppose $2, \delta, 2 \delta \notin E^{* 2}$, then $\widetilde{\Delta}_{E}=\left\{2, \delta, \delta^{\prime}\right\} / E^{* 2}$. Although $\Delta_{E} \subset D(E / F)$, it is difficult to find $\delta^{\prime}$ from the representative set of $D(E / F)$ in Theorem 2.1 .

About $T$ in Theorem 2.1 (4), we have the following result.

Proposition 2.3 Let $-d_{1} \equiv 1 \bmod 8,2 \in N F$ and $u^{2}-2 w^{2}=-d_{1}, u, w \in \mathbb{N}$. Suppose $m\left(u+\sqrt{-d_{1}}\right) \in N_{E / F}\left(E^{*}\right) \cap \Delta_{F}, m \mid d_{1}$, then

(1) $q_{j} \equiv \pm 1 \bmod 8$ for each odd prime divisor $q_{j}$ of $d_{2}$.

(2) If $-d_{1} \equiv 9 \bmod 16$, then $d_{2} \equiv \pm 1 \bmod 8$.

Proof. Let $u^{2}-2 w^{2}=-d_{1}, u, w \in \mathbb{N}, w \equiv 4 \bmod 8$. Suppose $m\left(u+\sqrt{-d_{1}}\right) \in N_{E / F}\left(E^{*}\right) \cap \Delta_{F}$. By $m\left(u+\sqrt{-d_{1}}\right) \in \Delta_{F}$, in the field $F$ the Hilbert symbol $\left(m\left(u+\sqrt{-d_{1}}\right),-1\right)_{2}=1$ and $m+u \equiv 2 \bmod 4$, e.g. see [14]. By $m\left(u+\sqrt{-d_{1}}\right) \in N_{E / F}\left(E^{*}\right)$, in the field $F$ the Hilbert symbol $\left(m\left(u+\sqrt{-d_{1}}\right), d_{2}\right)_{2}=1$ and $\left(m\left(u-\sqrt{-d_{1}}\right), d_{2}\right)_{2}=1$. Hence $\left(2 m^{2} w^{2}, d_{2}\right)_{2}=\left(2, d_{2}\right)_{2}=1$, so the local Hilbert symbol $\left(\frac{2, d_{2}}{Q_{j}}\right)=\left(\frac{2}{q_{j}}\right)=1$ for each odd prime $Q_{j} \mid q_{j}$ of $F$. Hence $q_{j} \equiv \pm 1 \bmod 8$ for each $q_{j} \mid d_{2}$.

If $-d_{1} \equiv 9 \bmod 16$, then $u \equiv \pm 3 \bmod 8$. By $m+u \equiv 2 \bmod 4$ and $m \mid d_{1}$, i.e. $m \equiv \pm 1 \bmod 8$,

$$
m \frac{u+\sqrt{-d_{1}}}{2} \equiv m u \equiv 5 \bmod \left(2, \frac{u-\sqrt{-d_{1}}}{2}\right)^{3}=\mathcal{D}^{3} .
$$

Suppose that $m\left(u+\sqrt{-d_{1}}\right) \in N_{E / F}\left(E^{*}\right)$, then in the complete field $F_{\mathcal{D}}, \mathcal{D} \mid 2$, the local Hilbert symbol

$$
\left(\frac{m\left(u+\sqrt{-d_{1}}\right), d_{2}}{\mathcal{D}}\right)=\left(\frac{5, d_{2}}{\mathcal{D}}\right)=1 .
$$

Hence $d_{2} \equiv \pm 1 \bmod 8$.

Although we know the forms of all elements of order $\leq 2$ in $K_{2} O_{E} \cap \operatorname{Tr}(E / F)$ from Theorem 2.1, we are interested in the forms of all elements of order $\leq 2$ in $\mathrm{K}_{2} \mathrm{O}_{E}$.

Since $C^{S}(F)$ has odd order, we use the method of proving [5, proposition 2.1] to get the following result.

Lemma 2.1 If $x \in N_{E / F}\left(E^{*}\right)$, then $x \in D_{F}$ if and only if there is $\alpha \in D_{E}$ with $N_{E / F}(\alpha)=$ $x f^{2}, f \in F^{*}$.

Proof. We only need to prove the necessary condition. Suppose $N_{E / F}\left(\alpha^{\prime}\right)=x, \alpha^{\prime} \in E$, $x \in D_{F}$, then the valuation of $\alpha^{\prime}$ is even at all undecomposed non-dyadic finite primes and $v_{\mathcal{P}}\left(\alpha^{\prime}\right)=v_{\mathcal{P}^{\sigma}}\left(\alpha^{\prime}\right) \bmod 2$ at finite decomposed primes $P O_{E}=\mathcal{P} \mathcal{P}^{\sigma}$. Since $C^{S}(F)$ has odd order, we find $f \in F^{*}$ such that $\alpha=\alpha^{\prime} f \in D_{E}$ and $N_{E / F}(\alpha)=x f^{2}$.

Let $F=\mathbb{Q}\left(\sqrt{-d_{1}}\right)$ and $E=F\left(\sqrt{d_{2}}\right)$. Suppose $m$ is a positive divisor of $2 d_{1}$. We have that $m \in N_{E / F}\left(E^{*}\right)$ 
$\Longleftrightarrow$ the Hilbert symbol $\left(m, d_{2}\right)_{2}=1$ of $F$

$\Longleftrightarrow$ the local Hilbert symbol $\left(\frac{m, d_{2}}{P}\right)=1$ for each primes $P$ of $F$.

By $[8$, theorem 5.4], for each finite non-dyadic prime $P$,

$$
\left(\frac{m, d_{2}}{P}\right)=\left((-1)^{v_{P}(m) v_{P}\left(d_{2}\right)} m^{v_{P}\left(d_{2}\right)} d_{2}^{-v_{P}(m)}\right)^{(N P-1) / 2} \bmod \mathrm{P} .
$$

Hence, for each finite prime $P \notin S,\left(\frac{m, d_{2}}{P}\right)=1$ and, for each finite non-dyadic prime $Q \in S$, $\left(\frac{m, d_{2}}{Q}\right)=\left(\frac{m}{Q}\right)=\left(\frac{m}{q}\right)$, where $Q$ is a prime over $q$. In the following, we find out all $m$ with $\left(\frac{m, d_{2}}{Q}\right)=\left(\frac{m}{q}\right)=1$ for all $Q \in S_{f}, Q \mid q$.

If $2 \mid D_{F}$, i.e., $p_{t}=2$. We define the system of homogeneous equations over $\mathbb{F}_{2}$

$$
A X=0
$$

where

$$
A=\left(\begin{array}{lll}
\left(\frac{p_{1}}{q_{1}}\right)^{\prime} & \cdots & \left(\frac{p_{t}}{q_{1}}\right)^{\prime} \\
\vdots & & \vdots \\
\left(\frac{p_{1}}{q_{s+r}}\right)^{\prime} & \cdots & \left(\frac{p_{t}}{q_{s+r}}\right)^{\prime}
\end{array}\right)
$$

Let a non-zero vector $X=\left(x_{1}, \cdots, x_{t}\right)^{T}$ over $\mathbb{F}_{2}$ be corresponding to $m=\prod_{x_{i}=1} p_{i}$. Hence each non-zero solution of the system (2.9) is 1-1 corresponding to $m \in N_{E / F}\left(E^{*}\right)$.

If $2 \nmid D_{F}$, i.e., $p_{t} \neq 2$. We also define the system of homogeneous equations over $\mathbb{F}_{2}$

$$
A^{\prime} X=0
$$

where

$$
A^{\prime}=\left(\begin{array}{llll}
\left(\frac{2}{q_{1}}\right)^{\prime} & \left(\frac{p_{1}}{q_{1}}\right)^{\prime} & \ldots & \left(\frac{p_{t}}{q_{1}}\right)^{\prime} \\
\vdots & \vdots & & \vdots \\
\left(\frac{2}{q_{s+r}}\right)^{\prime} & \left(\frac{p_{1}}{q_{s+r}}\right)^{\prime} & \ldots & \left(\frac{p_{t}}{q_{s+r}}\right)^{\prime} \\
\left(\frac{2, d_{2}}{\mathcal{D}}\right)^{\prime} & \left(\frac{p_{1}, d_{2}}{\mathcal{D}}\right)^{\prime} & \ldots & \left(\frac{p_{t}, d_{2}}{\mathcal{D}}\right)^{\prime}
\end{array}\right)
$$

where $\mathcal{D}$ is a dyadic prime of $F$ ( in fact, we need the last row of $A^{\prime}$ only if $d_{1} \equiv-1 \bmod$ $8)$. Hence each non-zero solution of the system $\left(2.10^{\prime}\right)$ is $1-1$ corresponding to $m \in N_{E / F}\left(E^{*}\right)$. Hence we get:

Lemma 2.2 If $2 \mid D_{F}$, then there is a non-zero solution $X=\left(x_{1}, \cdots, x_{t}\right)^{T}$ of (2.9) if and only if $m \in N_{E / F}\left(E^{*}\right)$, where $m=\prod_{x_{i}=1} p_{i}$; if $2 \nmid D_{F}$ and $p_{0}=2$, then there is a non-zero solution $X=\left(x_{0}, x_{1}, \cdots, x_{t}\right)^{T}$ of (2.9') if and only if $m \in N_{E / F}\left(E^{*}\right)$, where $m=\prod_{x_{i}=1} p_{i}$.

Theorem 2.2 Suppose $A$ and $A^{\prime}$ are defined as (2.10) and (2.10'), respectively. If $2 \mid D_{F}$, then

$$
r_{2}\left(K_{2} O_{E}\right)=2 s+r+s_{2}+t-3-\operatorname{rank} A
$$


if $2 \nmid D_{F}$, then

$$
r_{2}\left(K_{2} O_{E}\right)=2 s+r+s_{2}+t-2-\operatorname{rank} A^{\prime}+\varepsilon,
$$

where $\varepsilon=1$ if $m\left(u+\sqrt{-d_{1}}\right) \in N_{E / F}\left(E^{*}\right), u^{2}-2 w^{2}=-d_{1}$, some $m \mid 2 d_{1}$, and $\varepsilon=0$ otherwise.

Proof. Since $F=\mathbb{Q}\left(\sqrt{-d_{1}}\right)$ and $E=\mathbb{Q}\left(\sqrt{-d_{1}}, \sqrt{d_{2}}\right)$, by [5] there is a commutative diagram

$$
\begin{aligned}
& 0 \rightarrow K_{2} O_{E} \rightarrow K_{2} E \rightarrow \quad \bigoplus \bar{E}_{v} \rightarrow 0 \\
& \downarrow T r \quad \downarrow T r \quad v \text { fin. } \\
& 0 \rightarrow K_{2} O_{F} \rightarrow K_{2} F \rightarrow \underset{v \text { fin. }}{\bigoplus} \bar{F}_{v} \rightarrow 0 .
\end{aligned}
$$

Hence there is a restrictive homomorphism of two elementary subgroups $\operatorname{Tr}:{ }_{2} \mathrm{~K}_{2} \mathrm{O}_{E} \rightarrow$ ${ }_{2} \mathrm{~K}_{2} \mathrm{O}_{F}$. Set $\overline{\mathrm{H}}=\operatorname{Tr}\left({ }_{2} \mathrm{~K}_{2} \mathrm{O}_{\mathrm{E}}\right)$, then there is an exact sequence

$$
0 \rightarrow{ }_{2}\left(K_{2} O_{E} \cap \operatorname{Tr}(E / F)\right) \rightarrow{ }_{2} K_{2} O_{E} \stackrel{\operatorname{Tr}}{\rightarrow} \bar{H} \rightarrow 0 .
$$

Therefore we have

$$
{ }_{2} K_{2} O_{E}={ }_{2}\left(K_{2} O_{E} \cap \operatorname{Tr}(E / F)\right) \oplus H, \operatorname{Tr}(H)=\bar{H} .
$$

By (2.6), we only need to computer $r_{2}(H)$ in the following two cases.

Case 1: $2 \notin N F$. By [1], all elements of order $\leq 2$ in $K_{2} O_{F}$ are the forms: $\{-1, m\}$, where $m$ are all positive divisors of $2 d_{1}$, and $\Delta_{F}=\left\{2, m^{\prime}\right\} F^{* 2}$, where $m^{\prime}$ is a positive odd divisor of $d_{1}$.

If $2 \mid D_{F}$, i.e. $p_{t}=2$, then, by Lemma 2.1 and 2.2 , there is $\beta \in H$ with $\operatorname{Tr}(\beta)=\{-1, m\} \in \bar{H}$ if and only if $m \in N_{E / F}\left(E^{*}\right)$ if and only if there is a non-zero solution of (2.9) corresponding to $m$. By the Tate kernel $\Delta_{F}=\left\{2, m^{\prime}\right\} F^{* 2}$ and the definition of $\epsilon$ in (2.3), we get

$$
\begin{gathered}
r_{2}(H)=t-\operatorname{rank} A-(2-\epsilon), \\
r_{2}\left(K_{2} O_{E}\right)=2 s+r+s_{2}+t-3-\operatorname{rank} A
\end{gathered}
$$

If $2 \nmid D_{F}$ and $2 \notin N F$, then, similarly, we get

$$
\begin{gathered}
r_{2}(H)=t+1-\operatorname{rank} A^{\prime}-(2-\epsilon), \\
r_{2}\left(K_{2} O_{E}\right)=2 s+r+s_{2}+t-2-\operatorname{rank} A^{\prime} .
\end{gathered}
$$

Moreover, let $\Delta_{F}=\left\{2, m^{\prime}\right\} F^{* 2}$, we can determine the value of $\epsilon$ from the system (2.9) or (2.9'). In fact, $m^{\prime} \in N_{E / F}\left(E^{*}\right) \cap \Delta_{E}$ if and only if the column vector $X$ corresponding to $m^{\prime}$ must be a solution of $(2.9)$ or $\left(2.9^{\prime}\right)$.

Case 2: $d_{1} \equiv-1 \bmod 8$ and $2 \in N F$. By [1] all elements of order $\leq 2$ in $K_{2} O_{F}$ are the forms: $\{-1, m\},\{-1, m(u+\sqrt{d})\}$, where $m$ are all positive divisors of $d_{1}$ and $u^{2}-2 w^{2}=-d_{1}, u, w \in \mathbb{N}$; by Proposition $2.1 \Delta_{F}=\left\{2, m^{\prime}(u+\sqrt{d})\right\} F^{* 2}$, where $m^{\prime}$ is a positive odd divisor of $d_{1}$. 
First, suppose $m\left(u+\sqrt{-d_{1}}\right) \notin N_{E / F}\left(E^{*}\right)$ for each $m \mid 2 d_{1}$, then, by the preceding discussion, we get the same results as case 1. E.g., if there is some $q_{i} \equiv \pm 3 \bmod 8$, then $m\left(u+\sqrt{-d_{1}}\right) \notin$ $N_{E / F}\left(E^{*}\right)$ for each such $m$.

On the other hand, suppose $m^{\prime}\left(u+\sqrt{-d_{1}}\right) \in N_{E / F}\left(E^{*}\right)$, then, by Proposition 2.2 each prime $q_{i} \equiv \pm 1 \bmod 8$ and $\epsilon=0$ in (2.3). By the preceding discussion, we get

$$
r_{2}(H)=t+1-\operatorname{rank} A^{\prime}-1 .
$$

Suppose $\epsilon=1$ and $m^{\prime \prime}\left(u+\sqrt{-d_{1}}\right) \in N_{E / F}\left(E^{*}\right)$ for some $m^{\prime \prime} \neq m^{\prime}, m^{\prime \prime} \mid 2 d_{1}$, then $2 \in N_{E / F}\left(E^{*}\right)$ by Proposition 2.2 and

$$
r_{2}(H)=t+1-\operatorname{rank} A^{\prime}-1+1 .
$$

In the above two cases, we always have

$$
r_{2}\left(K_{2} O_{E}\right)=2 s+r+s_{2}+t-2-\operatorname{rank} A^{\prime}+\varepsilon, \varepsilon=1 .
$$

We prove Theorem 2.2 by the method of construction, hence we can represent all elements of order $\leq 2$ in $\mathrm{K}_{2} \mathrm{O}_{E}$.

Example 2.1 $F=\mathbb{Q}(\sqrt{-3 \times 7}), E=\mathbb{Q}(\sqrt{-3 \times 7}, \sqrt{5 \times 11 \times 13})$.

For $F=\mathbb{Q}(\sqrt{-3 \cdot 7})$, set $p_{1}=3, p_{2}=7, p_{3}=2$. There is the Rédei's matrix

$$
M_{F}^{\prime}=\left(\begin{array}{lll}
\left(\frac{D / p_{1}^{*}}{p_{1}}\right)^{\prime} & \left(\frac{p_{2}}{p_{1}}\right)^{\prime} & \left(\frac{p_{3}}{p_{1}}\right)^{\prime} \\
\left(\frac{p_{1}}{p_{2}}\right)^{\prime} & \left(\frac{D / p_{2}^{*}}{p_{2}}\right)^{\prime} & \left(\frac{p_{3}}{p_{2}}\right)^{\prime}
\end{array}\right)=\left(\begin{array}{lll}
\left(\frac{-7}{3}\right)^{\prime} & \left(\frac{7}{3}\right)^{\prime} & \left(\frac{2}{3}\right)^{\prime} \\
\left(\frac{3}{7}\right)^{\prime} & \left(\frac{-3}{7}\right)^{\prime} & \left(\frac{2}{7}\right)^{\prime}
\end{array}\right)=\left(\begin{array}{ccc}
1 & 0 & 1 \\
1 & 1 & 0
\end{array}\right) .
$$

Hence $r_{4}(C(F))=0$. It is clear that $\Delta_{F}=\{2,7\} F^{* 2}$. On the other hand, 5,11 are decomposable in $F$ and 13 is inert in $F$. Set $q_{1}=5, q_{2}=11, q_{3}=13$, there is a matrix as $(2.2)$

$$
M^{S}=\left(\begin{array}{ccc}
\left(\frac{2}{p_{1}}\right)^{\prime} & \left(\frac{q_{1}}{p_{1}}\right)^{\prime} & \left(\frac{q_{2}}{p_{1}}\right)^{\prime} \\
\left(\frac{2}{p_{2}}\right) & \left(\frac{q_{1}}{p_{2}}\right)^{\prime} & \left(\frac{q_{2}}{p_{2}}\right)^{\prime}
\end{array}\right)=\left(\begin{array}{ccc}
1 & 1 & 1 \\
0 & 1 & 0
\end{array}\right) .
$$

Since $\operatorname{rank} M^{S}=2, C^{S}(F)$ has odd order. We also have a matrix as (2.10)

$$
A=\left(\begin{array}{ccc}
\left(\frac{p_{1}}{q_{1}}\right)^{\prime} & \left(\frac{p_{2}}{q_{1}}\right)^{\prime} & \left(\frac{p_{3}}{q_{1}}\right)^{\prime} \\
\left(\frac{p_{1}}{q_{2}}\right)^{\prime} & \left(\frac{p_{2}}{q_{2}}\right)^{\prime} & \left(\frac{p_{3}}{q_{2}}\right)^{\prime} \\
\left(\frac{p_{1}}{q_{3}}\right)^{\prime} & \left(\frac{p_{2}}{q_{3}}\right)^{\prime} & \left(\frac{p_{3}}{q_{3}}\right)^{\prime}
\end{array}\right)=\left(\begin{array}{ccc}
1 & 1 & 1 \\
0 & 1 & 1 \\
0 & 1 & 1
\end{array}\right) .
$$

Hence $\operatorname{rank} A=2,2 \cdot 7 \in N_{E / F}\left(E^{*}\right) \cap \Delta_{E}$ and $r_{2}(H)=0$ in (2.11), so $r_{2}\left(K_{2} O_{E}\right)=2 s+r+$ $s_{2}+t-3-\operatorname{rank} A=4$. By Theorem 2.1 and Theorem 2.2, we have the representative set of $D(E / F) / E^{* 2}$ :

$$
\{3,7,2,11,13,1+\sqrt{-3 \cdot 7}\} \cup\{27+\sqrt{5 \cdot 11 \cdot 13}\}
$$

where $2 \cdot 11 \cdot 1^{2}=1^{2}+3 \cdot 7 \cdot 1^{2}$ and $2 \cdot 7 \cdot 1^{2}=27^{2}-5 \cdot 11 \cdot 13 \cdot 1^{2}$. Since $2,7 \in \Delta_{F} \subset \Delta_{E}$, the representative set of ${ }_{2} \mathrm{~K}_{2} \mathrm{O}_{\mathrm{E}}$ is

$$
\{-1,3\},\{-1,11\},\{-1,13\},\{-1,1+\sqrt{-21}\},\{-1,27+\sqrt{715}\}
$$

where there is only one dependent relation. 
Example 2.2 $F=\mathbb{Q}(\sqrt{-23}), E=\mathbb{Q}(\sqrt{-23}, \sqrt{47})$.

It is clear that $r_{2}(C(F))=0$ and $C^{S}(F)$ has odd order. Since $-23=3^{2}-2 \cdot 4^{2}, \Delta_{F}=\{2,23(3+$ $\sqrt{-23})\} F^{* 2}$. Also $20^{2}+23 \cdot 1^{2}=47 \cdot 3^{2}, P=(20+\sqrt{-23}, 47)$ and $P^{\prime}=(20-\sqrt{-23}, 47)$ are prime ideals of $F$ over 47 , then the local Hilbert symbol $\left(\frac{23(3+\sqrt{-23}), 47}{P}\right)=1$. Hence $2,23(3+\sqrt{-23}) \in$ $\Delta_{F} \cap N_{E / F}\left(E^{*}\right)$, so $N_{E / F}(7+\sqrt{47})=2$ and $N_{E / F}(\alpha)=23(3+\sqrt{-23}) f^{2}$ for $\alpha \in D(E / F)$. By Theorem 2.1 and Theorem 2.2, $r_{2}\left(K_{2} O_{E} \cap \operatorname{Tr}(E / F)\right)=2+0+2-1-\epsilon=3, r_{2}(H)=0$ in (2.11) and the representative set of ${ }_{2} K_{2} O_{E}$ is

$$
\{-1,23\},\{-1,20+\sqrt{-23}\},\{-1,7+\sqrt{47}\},\{-1, \alpha\}
$$

where there is only one dependent relation.

\section{4-rank}

Let $F=\mathbb{Q}\left(\sqrt{-d_{1}}\right)$ and $E=\mathbb{Q}\left(\sqrt{-d_{1}}, \sqrt{d_{2}}\right)$ be an imaginary quadratic field and a biquadratic field, respectively. Let $S$ be the finite set of primes in $F$ consisting of all infinite primes, all dyadic primes, all finite primes ramifying in $E$. In this section, we will use the Hurrelbrink and Kolsters' results in [5] to investigate elements of order 4 of $\mathrm{K}_{2} \mathrm{O}_{E}$, provided the 4-rank of the class group $C(F)$ is 0 and the $S$-ideal class group $C^{S}(F)$ has odd order.

First, we describe those results of [5]. Let $\operatorname{Tr}(E / F)$ is the kernel of a homomorphism $\operatorname{Tr}: K_{2} E \rightarrow K_{2} F$ and let $\gamma=\frac{\alpha}{\alpha^{\sigma}} \in \operatorname{Tr}(E / F)$, then we define a homomorphism

$$
\begin{aligned}
\phi_{E / F}: \operatorname{Tr}(E / F) & \rightarrow K_{2} F /\left\{-d_{2}, \Delta_{F}\right\} \cdot\left(K_{2} F\right)^{2} \cong{ }_{2} B r(F) /\left(-d_{2}, \Delta_{F}\right) \\
\gamma & \mapsto \operatorname{Tr}(\alpha) \bmod \left\{-d_{2}, \Delta_{F}\right\} \cdot\left(K_{2} F\right)^{2}
\end{aligned}
$$

where $\left(-d_{2}, \Delta_{F}\right)$ denotes the subgroup of the Brauer group of $\mathrm{F}$ generated by the classes $\left(-d_{2}, \varepsilon\right), \varepsilon \in \Delta_{F}$. Suppose $C^{S}(F)$ has odd order, there are exact sequences in [5, proposition 2.10 , corollary 2.12]

$$
\begin{gathered}
0 \rightarrow \operatorname{Tr}(E / F)^{2} \rightarrow \operatorname{Tr}(E / F) \stackrel{\phi_{E / F}}{\rightarrow} \operatorname{Br}(F) /\left(-d_{2}, \Delta_{F}\right) \\
0 \rightarrow\left(K_{2} O_{E}\right)^{2} \cap \operatorname{Tr}(E / F) \rightarrow K_{2} O_{F} \cap \operatorname{Tr}(E / F) \stackrel{\phi_{E / F}}{\rightarrow}{ }_{2} B r^{S}(F) /\left(-d_{2}, \Delta_{F}\right) .
\end{gathered}
$$

If $\gamma=\{-1, t\} \in \operatorname{Tr}(E / F), t \in F^{*}$, then $\phi_{E / F}(\gamma)=\overline{\left(-d_{2}, t\right)}$ by [5, proposition 2.13].

To compute the homomorphism $\phi_{E / F}$ easier, we define another homomorphism in [5]. Let $E_{1}=E=F\left(\sqrt{d_{2}}\right) \neq E_{2}=F(i), E_{3}=E_{0}=F\left(\sqrt{-d_{2}}\right)$. Suppose $x \in R(E / F)=N_{E / F}^{-1}\left(\Delta_{F}\right)$ and $y \in F(i)$, satisfying $N_{E / F}(x)=N_{F(i) / F}(y)$. We call $y$ admissible for $x$ if

$$
\operatorname{Tr}_{F(i) / F}(\{i, y\})=1 \text { and } t_{E / F}(x)+t_{F(i) / F}(y) \neq 0,
$$

where $t_{E / F}$ and $t_{F(i) / F}$ denote the trace maps from $E$ and $F(i)$ to $F$, respectively. In this case we also call $(x, y)$ an admissible pair. Moreover, provided that each element $x \in R(E / F)$ has 
an admissible element $y$, we define the homomorphism

$$
\begin{aligned}
\psi_{E / F}:{ }_{2} \operatorname{Tr}(E / F) & \rightarrow \operatorname{Br}\left(E_{0} / F\right) /\left(-d_{2}, \Delta_{F}\right), \\
\{-1, x\} & \mapsto\left(-d_{2}, t_{E / F}(x)+t_{F(i) / F}(y)\right),
\end{aligned}
$$

where $\operatorname{Br}\left(E_{0} / F\right)$ consists of all classes of quaternion algebras of the form $\left(-d_{2}, a\right)$ with $a \in F^{*}$. Hence, by [5, theorem 3.7 and corollary 3.9] and $D(E / F)=R(E / F) \cap D_{E}$ in section 2, there is a result:

Lemma 3.1 Suppose $C^{S}(F)$ has odd and each element $x \in D(E / F)$ has an admissible element $y \in F(i)$. Then there is a exact sequence

$$
0 \rightarrow{ }_{2} K_{2} O_{E} \cap \operatorname{Tr}(E / F) \cap K_{2} O_{E}^{2} \rightarrow{ }_{2} K_{2} O_{E} \cap \operatorname{Tr}(E / F) \stackrel{\psi_{E / F}}{\rightarrow}{ }_{2} B r^{S}\left(E_{0} / F\right) /\left(-d_{2}, \Delta_{F}\right) .
$$

In the section, we will use Lemma 3.1 to compute the 4-rank of $\mathrm{K}_{2} \mathrm{O}_{E}$. Hence we need to investigate the condition of Lemma 3.1. In fact, if $\phi_{F(i) / F}$ is trivial, then there is always an admissible $y \in F(i)$ for $x \in R(E / F)$; specially, if $K_{2} O_{F}^{\prime}(2)=0$, then ${ }_{2} \phi_{F(i) / F}$ is trivial, compare e.g. [2, proposition 2.17].

Let $F=\mathbb{Q}\left(\sqrt{-d_{1}}\right)$ and $E=\mathbb{Q}\left(\sqrt{-d_{1}}, \sqrt{d_{2}}\right)$ be an imaginary quadratic field and a biquadratic field, respectively. We have a quite general result:

Proposition 3.1 Suppose that $r_{4}(C(F))=0,2 \notin N F$ and $C^{S}(F)$ has odd order. Then each element $x \in D(E / F)$ has an admissible element $y \in F(i)$.

Proof. By Proposition 2.1, the Tate kernel $\Delta_{F}=\{2, m\} F^{* 2}, m \mid d_{1}$. If $x \in D(E / F)$ and $N_{E / F}(x)=2 \in \Delta_{F}$, then $y=1+i$ or $-1+i \in F(i)$ satisfies $\operatorname{Tr}_{F(i) / F}(\{i, y\})=1$ and $t_{E / F}(x)+t_{F(i) / F}(y) \neq 0$, so $y$ is admissible for $x$. We only need to find an admissible element $y$ for each element $x \in D(E / F), N_{E / F}(x)=m$ or $2 m \in \Delta_{F}$.

Case 1: $d_{1} \not \equiv \pm 1 \bmod 8$. By $r_{4}(C(F))=0$, Proposition 2.1 and [17, theorem 4.3], $r_{4}\left(K_{2} O_{F}\right)=$ $0, \Delta_{F}=\{2, m\} F^{* 2}$ and $\left[P_{m}\right]=1$ in the narrow class group $C\left(F_{0}\right)$ of $F_{0}=\mathbb{Q}\left(\sqrt{d_{1}}\right)$. Without loss of generality, we assume that there are integers $a, b \in \mathbb{N}$ such that

$$
m=a^{2}-d_{1} b^{2}
$$

Take $\alpha=a+\left(-\sqrt{-d_{1}} b\right) i \in F(i)$, then

$$
\operatorname{Tr}_{F(i) / F}\{i, \alpha\}=\operatorname{Tr}_{F(i) / F}\left\{\alpha / a, \sqrt{-d_{1}} b / a\right\}=\left\{m / a^{2}, \sqrt{-d_{1}} b / a\right\}
$$

Note that $a^{2}-d_{1} b^{2}=m \in \Delta_{F}$ and $\operatorname{Tr}_{F(i) / F}\{i, \alpha\}^{2}=\{-1, m\}=1$, then

$$
\beta=\left\{\frac{m}{a^{2}}, \frac{\sqrt{-d_{1}} b}{a}\right\}=\{-1, t\} \in K_{2} O_{F}
$$


By $d_{1} \not \equiv \pm 1 \bmod 8$ and $F$ imaginary,

$$
\beta \in K_{2} F^{2} .
$$

Hence there is an element $\alpha^{\prime} \in F(i)$ such that $t=N_{F(i) / F}\left(\alpha^{\prime}\right)$. We get such $y=t^{-1} \alpha \alpha^{\prime 2} \in F(i)$ with $\operatorname{Tr}_{F(i) / F}(\{i, y\})=1$ and $N_{F(i) / F}(y)=m$. Hence $(x, y)$ is an admissible pair.

Case 2: $d_{1} \equiv 1 \bmod 8$. By $r_{4}(C(F))=0$ and the process of proving Proposition 2.1 (2), we get that $r_{4}\left(C\left(F_{0}\right)\right)=0$ and the ambiguous class $\left[P_{n}\right]=1$ in $C\left(F_{0}\right)$, where $C\left(F_{0}\right)$ is the narrow class group of $F_{0}=\mathbb{Q}\left(\sqrt{d_{1}}\right)$ and $n \equiv \pm 3 \bmod 8$ is a positive divisor of $d_{1}$. Hence, without loss of generality, $n=a^{2}-d_{1} b^{2}, a, b \in \mathbb{N}$. Since the rank of Rédei $t \times t$ matrix $M_{F_{0}}$ of $F_{0}$ is equal to $t-1$ and $d_{1}=p_{1} \cdots p_{t} \equiv 1 \bmod 8$, the system of equations

$$
M_{F_{0}} X=\left(\begin{array}{c}
\left(\frac{2}{p_{1}}\right)^{\prime} \\
\vdots \\
\left(\frac{2}{p_{t}}\right)^{\prime}
\end{array}\right)
$$

is solvable over $\mathbb{F}_{2}$. Hence there must be a positive divisor $m \equiv \pm 1 \bmod 8$ of $d_{1}$ such that the Diophantine equation

$$
2 m z^{2}=x^{2}-d_{1} y^{2}
$$

is solvable over $\mathbb{Z}$, see e.g. [17]. Hence $\{-1, m\} \in\left(K_{2} O_{F}\right)^{2}$, so $m \in \Delta_{F}$ by Proposition 2.1 (2). Moreover, the ideal class $[\mathcal{D}]\left[P_{m}\right] \in C\left(F_{0}\right)^{2}$, where $\mathcal{D}$ is a dyadic ideal and $P_{m}$ is an ambiguous ideal over $m$. Since $r_{4}\left(C\left(F_{0}\right)\right)=0$, $[\mathcal{D}]\left[P_{m}\right]$ must be of odd order $k$, so $\left([\mathcal{D}]\left[P_{m}\right]\right)^{k}=[\mathcal{D}]^{k}\left[P_{m}\right]=1$. Hence there are $a, b \in \mathbb{N}$ satisfying that

$$
2^{k} m=a^{2}-d_{1} b^{2}
$$

By the process of proving case 1, we also find $y \in F(i)$ satisfying $\operatorname{Tr}_{F(i) / F}(\{i, y\})=1$ and $N_{F(i) / F}(y)=m$. Hence $(x, y)$ is an admissible pair.

Case 3: $-d_{1} \equiv 1 \bmod 8$ with $2 \notin N F$. By Proposition $2.1 \Delta_{F}=\{2, m\} F^{* 2}$, where $m \equiv 1$ $\bmod 4$ is a positive divisor of $d_{1}$. In the following, we prove that $m, 2 m \notin N_{E / F}\left(E^{*}\right)$. We assume that primes $q_{0}=2, q_{1}, \cdots, q_{t-2}$ satisfy that $\operatorname{rank} M_{1}^{S}=t-1$ in (2.7). Set $Q_{i} \mid q_{i}, i=1, \cdots, t-2$.

Suppose that $m \in N_{E / F}\left(E^{*}\right)$, i.e., the Hilbert symbol $\left(m, d_{2}\right)_{2}=1$. By Minkowski-Hasse theorem, each local Hilbert symbol $\left(\frac{m, d_{2}}{Q_{i}}\right)=\left(\frac{m}{q_{i}}\right)=1, i=1, \cdots, t-2$. Since $m \equiv 1 \bmod 4$, each Jacobi symbol

$$
\left(\frac{q_{i}}{m}\right)=1, i=1, \cdots, t-2
$$

Set $m^{\prime}=d_{1} m \equiv-1 \bmod 4,1=\left(\frac{-d}{q_{i}}\right)=\left(\frac{m}{q_{i}}\right)\left(\frac{-m^{\prime}}{q_{i}}\right)=\left(\frac{-m^{\prime}}{q_{i}}\right), i=1, \cdots, t-2$, hence the Jacobi symbol

$$
\left(\frac{q_{i}}{m}\right)=1, i=1, \cdots, t-2 .
$$

Moreover, by $d_{1}=m m^{\prime} \equiv-1 \bmod 8$,

$$
\left(\frac{2}{m^{\prime}}\right)=\left(\frac{2}{m}\right) .
$$


It is contradictory to $\operatorname{rank} M_{1}^{S}=t-1$ in $(2.7)$.

Suppose that $2 m \in N_{E / F}\left(E^{*}\right)$, i.e., the Hilbert symbol $\left(2 m, d_{2}\right)_{2}=1$. the local Hilbert symbols $\left(\frac{2 m, d_{2}}{Q_{i}}\right)=\left(\frac{2 m}{q_{i}}\right)=1, i=1, \cdots, t-2$. Since $m \equiv 1 \bmod 4$, the Jacobi symbols

$$
\left(\frac{q_{i}}{m}\right)=\left(\frac{2}{q_{i}}\right), i=1, \cdots, t-2 .
$$

Set $m^{\prime}=d_{1} / m \equiv-1 \bmod 4,1=\left(\frac{-4 d_{1}}{q_{i}}\right)=\left(\frac{2 m}{q_{i}}\right)\left(\frac{-2 m^{\prime}}{q_{i}}\right)=\left(\frac{-2 m^{\prime}}{q_{i}}\right), i=1, \cdots, t-2$, hence the Jacobi symbol

$$
\left(\frac{q_{i}}{m^{\prime}}\right)=\left(\frac{2}{q_{i}}\right), i=1, \cdots, t-2 .
$$

Moreover, by $d_{1}=m \dot{m}^{\prime} \equiv-1 \bmod 8$,

$$
\left(\frac{2}{m}\right)=\left(\frac{2}{m^{\prime}}\right)
$$

It is also contradictory with $\operatorname{rank} M_{1}^{S}=t-1$ in $(2.7)$.

Therefore $m, 2 m \notin N_{E / F}\left(E^{*}\right)$, where $\Delta_{F}=\{2, m\} F^{* 2}$.

Proposition 3.2 Suppose that $r_{4}(C(F))=0, C^{S}(F)$ has odd order and $-d_{1} \equiv 1$ mod 8 with $2 \in N F$.

(1) If $-d_{1} \equiv 1 \bmod 16$, then each element $x \in D(E / F)$ has an admissible element $y \in F(i)$.

(2) If $-d_{1} \equiv 9 \bmod 16$ and $m\left(u+\sqrt{-d_{1}}\right) \in \Delta_{F} \cap N_{E / F}\left(E^{*}\right), m \mid d_{1}, u^{2}-2 w^{2} \in \Delta_{F}$, then there is not an admissible pair $(x, y)$ such that $N_{E / F}(x)=N_{F(i) / F}(y)=m\left(u+\sqrt{-d_{1}}\right), x \in D(E / F)$, $y \in F(i)$.

Proof. Since $r_{4}(C(F))=0$ and $-d_{1} \equiv 1 \bmod 8$ with $2 \in N F$, by Proposition $2.1 r_{4}\left(C\left(F_{0}\right)\right)=0$ for $F_{0}=\mathbb{Q}\left(\sqrt{d_{1}}\right)$ and $\Delta_{F}=\left\{2, m\left(u+\sqrt{-d_{1}}\right)\right\} F^{* 2}$, where $-d_{1}=u^{2}-2 w^{2}, u, w \in \mathbb{N}, m \mid d_{1}$, and $u+m \equiv 2 \bmod 4$. In the following, we will find an element $y \in F(i)$ such that $N_{F(i) / F}(y)=$ $m\left(u+\sqrt{-d_{1}}\right)$ and $\operatorname{Tr}_{E / F}(\{i, y\})=1$.

By $r_{4}\left(C\left(F_{0}\right)\right)=0$ and $2 \in N F$, the dyadic class $[\mathcal{D}]$ in $C\left(F_{0}\right)$ is trivial. Also $-d_{1}=u^{2}-2 w^{2}=$ $2(u+w)^{2}-(u+2 w)^{2}, u, w \in \mathbb{N}$, and

$$
2(u+w)\left(u+\sqrt{-d_{1}}\right)=w^{2}+\left(u+w+\sqrt{-d_{1}}\right)^{2} .
$$

Set $\alpha_{1}=w+\left(u+w+\sqrt{-d_{1}}\right) i=\left(u+2 w-\sqrt{d_{1}}\right)+(1+i)(u+w) \in F(i)$. In the field $F_{0}=\mathbb{Q}\left(\sqrt{d_{1}}\right)$, by $(u+2 w)^{2}-d_{1}=2(u+w)^{2}$

$$
\left(u+2 w-\sqrt{d_{1}}\right)=\mathcal{D} P_{u+w}^{2},
$$

where $\mathcal{D}$ is the dyadic ideal and $P_{u+w}$ is an integer ideal over $u+w$. Hence, in the class group $C\left(F_{0}\right),[\mathcal{D}]\left[P_{u+w}\right]^{2}=\left[P_{u+w}\right]^{2}=1$ and $\left[\bar{P}_{u+w}\right]^{2}=1$, where $\bar{P}_{u+w}$ is the conjugate ideal of $P_{u+w}$. Hence there is an ambiguous class $\left[P_{m}\right]$ such that $\left[P_{m}\right]\left[\bar{P}_{u+w}\right]=1$, so there is the integral element $\alpha_{2}=a+b \sqrt{d_{1}} \in O_{F_{0}}$ such that

$$
\left(a+b \sqrt{d_{1}}\right)=P_{m} \bar{P}_{u+w} \text { and } a^{2}-d_{1} b^{2}=m(u+w) .
$$


Set $\alpha=\alpha_{1} \alpha_{2}=e+f i \in F(i)$ and set $p$ an odd prime, $P$ a prime of $F$ over $p$ and $\mathcal{P}$ a prime of $F(i)$ over $P$. Then $v_{P}\left(N_{F(i) / F}(\alpha)\right) / 2+v_{\mathcal{P}}(\alpha) \equiv 0 \bmod 2$, for all odd primes $P$ of $F$. Thus, by [6, proposition 1.5] or [2],

$$
\begin{aligned}
\beta & =\operatorname{Tr}_{F(i) / F}\{i, \alpha\}=\left\{\frac{2 m\left(u+\sqrt{-d_{1}}\right)(u+w)^{2}}{e^{2}},-\frac{f}{e}\right\} \\
& =\operatorname{Tr}_{F(i) / F}\left\{i, \alpha_{1}\right\} \operatorname{Tr}_{F(i) / F}\left\{i, \alpha_{2}\right\} \\
& =\left\{\frac{2(u+w)\left(u+\sqrt{-d_{1}}\right)}{w^{2}},-\frac{u+w+\sqrt{-d_{1}}}{w}\right\}\left\{\frac{m(u+w)}{a^{2}},-\frac{\sqrt{-d_{1}} b}{a}\right\} \in K_{2} O_{F}, \\
\beta^{2} & =\left\{-1, m\left(u+\sqrt{-d_{1}}\right)\right\}=1,
\end{aligned}
$$

so

$$
\beta=\operatorname{Tr}_{F(i) / F}\{i, \alpha\}=\{-1, t\} \in K_{2} O_{F} .
$$

For all non-dyadic finite primes $P$, the local Hilbert symbol $\eta_{P}(\beta)=1$. Since $F$ has two dyadic primes $\mathcal{D}_{1}, \mathcal{D}_{2}, t \in N_{F(i) / F}\left(F(i)^{*}\right)$ if and only if the Hilbert symbol $\eta_{\mathcal{D}_{1}}(\beta)=1$. We need to compute the value of the local Hilbert symbol $\eta_{\mathcal{D}_{1}}(\beta)$ in a dyadic prime $\mathcal{D}_{1}$.

If $-d_{1} \equiv 1 \bmod 16$, then we assume that $-d_{1}=u^{2}-2 w^{2}, u, w \in \mathbb{N}, w \equiv 4 \bmod 8$, and $u \equiv \pm 1 \bmod 8($ see $[3,14])$. Set $\mathcal{D}_{1}=\left(2, \frac{u-\sqrt{-d_{1}}}{2}\right)$. Since $\frac{u-\sqrt{-d_{1}}}{2} \cdot \frac{u+\sqrt{-d_{1}}}{2}=\frac{w^{2}}{2} \equiv 0 \bmod 8$,

$$
\frac{u+\sqrt{-d_{1}}}{2}=u-\frac{u-\sqrt{-d_{1}}}{2} \equiv u \equiv \pm 1 \bmod \mathcal{D}_{1}^{3}
$$

and

$$
m \cdot \frac{u+\sqrt{-d_{1}}}{2} \equiv m u \equiv 1 \bmod \mathcal{D}_{1}^{3}
$$

by $m \cdot\left(u+\sqrt{-d_{1}}\right) \in \Delta_{F}, m+u \equiv 2 \bmod 4$. In the complete field $F_{\mathcal{D}_{1}} \cong \mathbb{Q}_{2}, N_{F(i) / F}(\alpha / 2)=$ $m \cdot \frac{u+\sqrt{-d_{1}}}{2} \cdot(u+w)^{2} \equiv 1 \bmod \mathcal{D}_{1}^{3}$. Hence

$$
\eta_{\mathcal{D}_{1}}(\operatorname{Tr}\{i, \alpha\})=\eta_{\mathcal{D}_{1}}\left(\left\{\frac{2 m\left(u+\sqrt{-d_{1}}\right)(u+w)^{2}}{e^{2}},-\frac{f}{e}\right\}\right)=1 .
$$

Therefore, in the case, $\beta=\{-1, t\} \in\left(K_{2} F\right)^{2}$, i.e., $t \in N_{F(i) / F}\left(F(i)^{*}\right)$. By the process of proving Proposition 3.1, we find $y \in F(i)$ such that $N_{F(i) / F}(y)=m\left(u+\sqrt{-d_{1}}\right)$ and $\operatorname{Tr}_{F(i) / F}\{i, y\}=1$. Therefore every element $x \in D(E / F)$ has an admissible element $y \in F(i)$.

If $-d_{1} \equiv 9 \bmod 16$, then we assume that $-d_{1}=u^{2}-2 w^{2}, u, w \in \mathbb{N}, w \equiv 4 \bmod 8$, and $u \equiv \pm 3 \bmod 8$. Hence

$$
m \cdot \frac{u+\sqrt{-d_{1}}}{2} \equiv m u \equiv 5 \bmod \mathcal{D}_{1}^{3}
$$

In the complete field $F_{\mathcal{D}_{1}} \cong \mathbb{Q}_{2}, m \cdot \frac{u+\sqrt{-d_{1}}}{2} \cdot(u+w)^{2} \equiv 5 \bmod \mathcal{D}_{1}^{3}$ and $\frac{f}{e} \equiv 2$ or $6 \bmod \mathcal{D}_{1}^{3}$ by $e^{2}+f^{2}=2 m\left(u+\sqrt{-d_{1}}\right)(u+w)^{2}$. By $[13$, p.252 table $]$

$$
\eta_{\mathcal{D}_{1}}(\operatorname{Tr}\{i, \alpha\})=\eta_{\mathcal{D}_{1}}\left(5,-\frac{e}{f}\right)=-1 .
$$

Hence $\beta=\operatorname{Tr}\{i, \alpha\} \notin\left(K_{2} F\right)^{2}$, so there is not any element $y \in F(i)$ such that $N_{F(i) / F}(y)=$ $m\left(u+\sqrt{-d_{1}}\right)$ and $\operatorname{Tr}\{i, y\}=1$.

In the following we compute 4-rank of $K_{2} O_{E}$ for the biquadratic field $E=\mathbb{Q}\left(\sqrt{-d_{1}}, \sqrt{d_{2}}\right)$. 
Theorem 3.1 Suppose $r_{4}(C(F))=0, C^{S}(F)$ have odd order and $d_{1} \not \equiv 1 \bmod 8$. Then $r_{4}\left(K_{2} O_{E}\right)=$ $r_{2}\left({ }_{2} K_{2} O_{E} \cap K_{2} O_{E}^{2} \cap \operatorname{Tr}(E / F)\right.$.

Proof. Since $r_{4}(C(F))=0$ and $d_{1} \not \equiv 1 \bmod 8, r_{4}\left(K_{2} O_{F}\right)=0$ by Proposition 2.1, i.e. $K_{2} O_{F}(2)$ is elementary. There is a homomorphism $\operatorname{Tr}: \mathrm{K}_{2} \mathrm{O}_{E}(2) \rightarrow \mathrm{K}_{2} \mathrm{O}_{F}(2)$ for two 2-Sylow subgroups, so there is an exact sequence

$$
0 \rightarrow K_{2} O_{E}(2) \cap \operatorname{Tr}(E / F) \rightarrow K_{2} O_{E}(2) \stackrel{\operatorname{Tr}}{\rightarrow} \operatorname{Tr}\left(K_{2} O_{E}\right)(2) \rightarrow 0 .
$$

By $\operatorname{Tr}\left(K_{2} O_{E}(2)\right) \subset K_{2} O_{F}(2)$ elementary, ${ }_{2} K_{2} O_{E} \cap K_{2} O_{E}^{2} \subset K_{2} O_{E}(2) \cap \operatorname{Tr}(E / F)$. Then

$$
r_{4}\left(K_{2} O_{E}\right)=r_{2}\left({ }_{2} K_{2} O_{E} \cap K_{2} O_{E}^{2}\right)=r_{2}\left({ }_{2} K_{2} O_{E} \cap K_{2} O_{E}^{2} \cap \operatorname{Tr}(E / F)\right) .
$$

Remark 3.1 If $d_{1} \equiv 1 \bmod 8$, then by Proposition 2.1 there is a positive divisor $n \equiv \pm 3 \bmod 8$ of $d_{1}$ such that $\beta \in K_{2} O_{F}, \beta^{2}=\{-1, n\} \neq 1$. Suppose there is not any $\beta \in K_{2} O_{E}$ of order 2 such that $\operatorname{Tr}(\beta)=\{-1, n\}$, then we get the same result as Theorem 3.1. Suppose there is $\beta \in K_{2} O_{E}$ of order 2 such that $\operatorname{Tr}(\beta)=\{-1, n\}$, then $\left.r_{4}\left(K_{2} O_{E}\right)-r_{2}\left({ }_{2} K_{2} O_{E} \cap K_{2} O_{E}^{2} \cap \operatorname{Tr}(E / F)\right)\right)=0$ or 1 . In fact, we can determine the above conditions by the method of proving Theorem 2.1.

By Proposition 3.1, Proposition 3.2, Theorem 3.1 and [5, corollary 3.9], we get

Theorem 3.2 Suppose $r_{4}(C(F))=0, C^{S}(F)$ has odd order and the assumption of Proposition 3.2(2) does not hold, then there is an exact sequence

$$
\begin{aligned}
0 \rightarrow{ }_{2} K_{2} O_{E} \cap K_{2} O_{E}^{2} \cap \operatorname{Tr}(E / F) & \rightarrow{ }_{2}\left(K_{2} O_{F} \cap \operatorname{Tr}(E / F)\right) \\
& \stackrel{\psi_{E / F}}{\rightarrow}{ }_{2} B r^{S}\left(E_{0} / F\right) /\left(-d_{2}, \Delta_{F}\right) .
\end{aligned}
$$

Moreover, if $d_{1} \not \equiv 1$ mod 8 , then

$$
r_{4}\left(K_{2} O_{E}\right)=2 s+r+s_{2}-1-\epsilon-r_{2}\left(\operatorname{Im} \psi_{E / F}\right) .
$$

It is clear that this result with the representative set of $R(E / F)$ and the action of $\psi_{E / F}$ yields a computational approach to the 4-rank of $\mathrm{K}_{2} \mathrm{O}_{E}$. For convenience, we describe the algorithm of $r_{4}\left(K_{2} O_{E}\right)$ in detail (see [5, P173]).

Let $Q_{0}, Q_{0}^{\prime}, Q_{1}, \cdots, Q_{s+r}$ denote the primes in $S_{f}$ with $Q_{0}, Q_{0}^{\prime}$ dyadic primes (note $Q_{0}=Q_{0}^{\prime}$ if $\left.d_{1} \not \equiv-1 \bmod 8\right)$. Let $\left[x_{1}\right],\left[x_{2}\right], \cdots,\left[x_{l}\right]$ denote a basis of the cokernel of the map

$$
\left(\Delta_{F} \cap N_{E / F}\left(E^{*}\right)\right) / F^{* 2} \rightarrow D(E / F) / E^{* 2} .
$$

Since $\left[d_{2}\right] \notin \Delta_{F} / F^{* 2}$, the above map is injective and $l=\left|S_{f}\right|=2 s+r+s_{2}$. We form the $\left(2 s+r+s_{2}\right) \times\left(2 s+r+s_{2}\right)$ matrix over $\mathbb{F}_{2}$ by replacing the 1's by 0's and the -1 's by 1 's

$$
M_{E / F}=\left(\left(-d_{2}, t_{E / F}\left(x_{i}\right)+t_{F(i) / F}\left(y_{i}\right)\right)_{Q_{j}}^{\prime}\right),
$$

where $y_{i} \in F(i)$ is admissible for $x_{i}$. By [5, lemma 3.8] the local Hilbert symbols $\left(-d_{2}, t_{E / F}\left(x_{i}\right)+\right.$ $\left.t_{E / F}\left(y_{i}\right)\right)_{P}=1$ for all finite primes $P \notin S$. Hence by the quadratic reciprocity law $\operatorname{rank} M_{E / F} \leq$ $2 s+r+s_{2}-1$. By the definition of $\epsilon$ and Theorem $3.2 r_{2}\left(\operatorname{Im} \psi_{E / F}\right)=\operatorname{rank} M_{E / F}-\epsilon$ and $r_{2}\left(K_{2} O_{E} \cap \operatorname{Tr}(E / F)\right)=\left|S_{f}\right|-1-\epsilon$. By Theorem 3.2 and [5, lemma 5.1], we get 
Corollary 3.1 The assumption is as Theorem 3.2.

(1) $r_{2}\left({ }_{2} K_{2} O_{E} \cap K_{2} O_{E}^{2} \cap \operatorname{Tr}(E / F)\right)=2 s+r+s_{2}-1-\operatorname{rank} M_{E / F}$.

(2)If $d_{1} \neq \equiv 1 \bmod 8$, then $r_{4}\left(K_{2} O_{E}\right)=2 s+r+s_{2}-1-\operatorname{rank} M_{E / F}$.

We now illustrate the method through some examples.

Example 3.1 Let $F=\mathbb{Q}(\sqrt{-3 \times 7}), E=\mathbb{Q}(\sqrt{-3 \times 7}, \sqrt{5 \times 11 \times 13})$. Describe the structure of the 2-Sylow subgroup of $\mathrm{K}_{2} \mathrm{O}_{E}$.

In Example 2.1, we know that $r_{4}(C(F))=0, C^{S}(F)$ has odd order, $\Delta_{F}=\{2,7\} F^{* 2}$ and the representative set of $D(E / F) / E^{* 2}$. Since 5,11 are decomposed in $F, 13$ is inert in $F, s=2$, $r=1, s_{2}=1$ and $2 \times 7 \in \Delta_{F} \cap N_{E / F}\left(E^{*}\right)$, let $x_{1}=2, x_{2}=3, x_{3}=11, x_{4}=13, x_{5}=$ $1+\sqrt{-21}, x_{6}=27+\sqrt{715}$ and let $Q_{0}\left|2, Q_{1}\right| 5, Q_{2}\left|5, Q_{3}\right| 11, Q_{4}\left|11, Q_{5}\right| 13$. There is the $6 \times 6$ matrix

$$
\begin{aligned}
M_{E / F}= & \left(\begin{array}{ccccccc}
\left(-d_{2}, x_{1}\right)_{Q_{0}}^{\prime} & \left(-d_{2}, x_{1}\right)_{Q_{1}}^{\prime} & * & \left(-d_{2}, x_{1}\right)_{Q_{3}}^{\prime} & * & \left(-d_{2}, x_{1}\right)_{Q_{5}}^{\prime} \\
\left(-d_{2}, x_{2}\right)_{Q_{0}}^{\prime} & \left(-d_{2}, x_{2}\right)_{Q_{1}}^{\prime} & * & \left(-d_{2}, x_{2}\right)_{Q_{3}}^{\prime} & * & \left(-d_{2}, x_{2}\right)_{Q_{5}}^{\prime} \\
\left(-d_{2}, x_{3}\right)_{Q_{0}}^{\prime} & \left(-d_{2}, x_{3}\right)_{Q_{1}}^{\prime} & * & \left(-d_{2}, x_{3}\right)_{Q_{3}}^{\prime} & * & \left(-d_{2}, x_{3}\right)_{Q_{5}}^{\prime} \\
\left(-d_{2}, x_{4}\right)_{Q_{0}}^{\prime} & \left(-d_{2}, x_{4}\right)_{Q_{1}}^{\prime} & * & \left(-d_{2}, x_{4}\right)_{Q_{3}}^{\prime} & * & \left(-d_{2}, x_{4}\right)_{Q_{5}}^{\prime} \\
\left(-d_{2}, x_{5}\right)_{Q_{0}}^{\prime} & \left(-d_{2}, x_{5}\right)_{Q_{1}}^{\prime} & * & \left(-d_{2}, x_{5}\right)_{Q_{3}}^{\prime} & * & \left(-d_{2}, x_{5}\right)_{Q_{5}}^{\prime} \\
\left(-d_{2}, x_{6}^{\prime}\right)_{Q_{0}}^{\prime} & \left(-d_{2}, x_{6}^{\prime}\right)_{Q_{1}}^{\prime} & * & \left(-d_{2}, x_{6}^{\prime}\right)_{Q_{3}}^{\prime} & * & \left(-d_{2}, x_{6}^{\prime}\right)_{Q_{5}}^{\prime}
\end{array}\right) \\
= & \left(\begin{array}{cccccc}
* & 1 & 1 & 1 & 1 & 1 \\
* & 1 & 1 & 0 & 0 & 0 \\
* & 0 & 0 & 1 & 1 & 1 \\
* & 1 & 1 & 1 & 1 & 0 \\
* & 0 & 1 & 1 & 1 & * \\
* & * & * & 0 & 1 & *
\end{array}\right) .
\end{aligned}
$$

In the following, we verify the value of $\left(-d_{2}, x_{i}\right)_{Q_{j}}$ of $M_{E / F}$.

(1) In the 5 -th row of $M_{E / F}$, since $\left(\frac{22}{5}\right)=-1,\left(-d_{2}, 1+\sqrt{-21}\right)_{Q_{3}}=1$ and $\left(-d_{2}, 1+\right.$ $\sqrt{-21})_{Q_{4}}=-1$. Note $\left(-d_{2}, 1+\sqrt{-21}\right)=(2 \cdot 5 \cdot 13 \cdot(1-\sqrt{-21}), 1+\sqrt{-21})$, we get other values.

(2) In the 6-th row of $M_{E / F}, x_{6}=27+\sqrt{715} \in E$ and $N_{E / F}\left(x_{6}\right)=14$, hence we need to find an $x_{6}$-admissible element $y \in F(i)$. Since $7 \cdot 2^{2}=7^{2}-21 \cdot 1^{2}$ and $\alpha=7 / 2+i \sqrt{-21} / 2 \in F(i)$, by $(3.12) \beta=\operatorname{Tr}\{i, \alpha\}=\{4 / 7, \sqrt{-21} / 7\}=\{28, \sqrt{-21}\} \in K_{2} O_{F}$ and $\beta^{2}=1$, in fact, $\beta=1$ (see [15]). Then $y=\alpha(-1-i)$ is admissible for $x_{6}$ and $x_{6}^{\prime}=t_{E / F}\left(x_{6}\right)+t_{E / F(i)}(y)=47-\sqrt{-21}$. Hence $N_{E / F}\left(x_{6}^{\prime}\right)=2230$ and $\left(\frac{2230}{11}\right)=-1$, so we get the values of the 6 -th row.

Therefore $\operatorname{rank} M_{E / F}=5$ and $r_{4}\left(K_{2} O_{F}\right)=0$. From the matrix $M_{E / F}$, we know that $\psi_{E / F}(\{-1,3 \cdot 11\})=1$, so $\Delta_{E}=\{2,7,-11\} E^{* 2}$.

By Proposition 3.2, we can not use our method to investigate the 4-rank of $K_{2} O_{E}$ in Example 2.2 .

Example 3.2 Let $F=\mathbb{Q}(\sqrt{-47}), E=\mathbb{Q}(\sqrt{-47}, \sqrt{-23})$. Describe the structure of the 2-Sylow subgroup of $\mathrm{K}_{2} \mathrm{O}_{E}$. 
It is clear that $r_{2}(C(F))=0, C^{S}(F)$ has odd order and 23 is inert in $F$. Since $-47=9^{2}-$ $2 \cdot 8^{2}, \Delta_{F}=\{2,9+\sqrt{-47}\} F^{* 2}$. By $20^{2}+23 \cdot 1^{2}=47 \cdot 3^{2},\{-1,20+\sqrt{-23}\} \in K_{2} O_{E}$ and $\operatorname{Tr}_{E / F}(\{-1,20+\sqrt{-23}\})=\{-1,-1\}$, so $r_{2}(H)=1$ in $(2.8)$ of Theorem 2.2. It is clear that $2,9+\sqrt{-47} \in \Delta_{F} \cap N_{E / F}\left(E^{*}\right)$, i.e, $N_{E / F}(3+\sqrt{-23})=2 \cdot 2^{4}$ and

$$
x=\frac{43-\sqrt{-47}}{12}+\frac{\sqrt{-23}(5+\sqrt{-47})}{12}, N_{E / F}(x)=9+\sqrt{-47} .
$$

By Theorem 2.1 and Theorem 2.2, $r_{2}\left(K_{2} O_{E} \cap \operatorname{Tr}(E / F)\right)=0+1+2-1-\epsilon=2, r_{2}\left(K_{2} O_{E}\right)=3$ and the representative set of ${ }_{2} \mathrm{~K}_{2} \mathrm{O}_{\mathrm{E}}$ is

$$
\{-1,47\},\{-1,3+\sqrt{-23}\},\{-1, x\},\{-1,20+\sqrt{-23}\}
$$

where there is only one dependent relation.

In the following, we use Theorem 3.2 to investigate the 4-rank of $K_{2} O_{E}$. Let $Q_{0}\left|2, Q_{0}^{\prime}\right| 2$ and $Q_{1} \mid 23$ and let $x_{1}=47, x_{2}=(3+\sqrt{-23}) / 4, x_{3}=x$. We know that $y_{2}=-1-i \in F(i)$ is $x_{2}$-admissible and we find an $x_{3}$-admissible element. By $2(9+\sqrt{-47})=8^{2}+(1+\sqrt{-47})^{2}$, Let $y_{3}^{\prime}=8+i(1+\sqrt{-47}) \in F(i)$, then

$$
\beta=\operatorname{Tr}\left\{i, y_{3}^{\prime}\right\}=\left\{\frac{2(9+\sqrt{-47})}{8^{2}},-\frac{1+\sqrt{-47}}{8}\right\}=\{1+\sqrt{-47}, 4\} .
$$

Since $\beta^{2}=1, \beta \in K_{2} O_{F}$ and $\beta \in K_{2} F^{2}, \beta=1$ by $r_{4}\left(K_{2} O_{F}\right)=0$ and $C(F)$ of odd order. Set $y_{3}=-y_{3}^{\prime}(1+i) / 2=-\frac{7-\sqrt{-47}}{2}-i \frac{9+\sqrt{-47}}{2} \in F(i)$, then $y_{3}$ is $x_{3}$-admissible. Hence $x_{2}^{\prime}=$ $t_{E / F}\left(x_{2}\right)+t_{E / F}\left(y_{2}\right)=\frac{-1}{2}$ and $x_{3}^{\prime}=t_{E / F}\left(x_{3}\right)+t_{F(i) / F}\left(y_{3}\right)=\frac{1+5 \sqrt{-47}}{6}$, so we get

$$
M_{E / F}=\left(\begin{array}{ccc}
1 & 1 & 0 \\
1 & 1 & 0 \\
0 & 1 & 1
\end{array}\right) .
$$

Hence $\operatorname{rank} M_{E / F}=2, r_{4}\left(K_{2} O_{E}\right)=0$ and $\Delta_{E}=\{2,-3-\sqrt{-23}, 9+\sqrt{-47}\} E^{* 2}$.

Acknowledgments. The research was partially supported by NNSF grant of China (10371054). 


\section{References}

[1] J.Browkin and A.Schinzel, On Sylow 2-subgroups of $\mathrm{K}_{2} \mathrm{O}_{F}$ for quadratic fields $F$, J. reine angew, Math.331 (1982) 101-113.

[2] P.E.Conner and J.Hurrelbrink, On the 4-rank of $\mathrm{K}_{2} \mathrm{O}_{F}$, Can. J. Math. 41 (1989), 932-960.

[3] P.E.Conner and J.Hurrelbrink, On the 4-rank of the tame kernel $K_{2}(\mathcal{O})$ in positive definite terms, J. of Number Th. 88, 263-282 (2001).

[4] E.Hecke, Lecture on the Theory of Algebraic Numbers, GTM 77, Springer-Verlag, 1981.

[5] J.Hurrelbrink and M.Kolster, Tame kernels under relative quadratic extensions and Hilbert symbols, J. reine angew. Math. 499(1998), 145-188.

[6] M.Kolster, The structure of the 2-Sylow subgroup of $K_{2}(O)$, I, Comment. Math. Helv. 61 (1986), 576-588.

[7] J.Milnor, Introduction to Algebraic K-theory, Ann. of Math. Stud. 72, Princeton Univ. Press, 1971.

[8] J.Neukirch, Class Field Theory, Springer, Berlin, 1986.

[9] H.Qin, The 2-Sylow subgroups of the tame kernel of imaginary quadratic fields, Acta Arith., 69 (1995), 153-169.

[10] -, The 4-rank of $\mathrm{K}_{2} \mathrm{O}_{F}$ for real quadratic fields, Acta Arith. 72 (1995), 323-333.

[11] J.Tate, Relations between $K_{2}$ and Galois cohomology, Invent. Math., 36(1976), 257-274.

[12] Tsuyoshi Uehara, On the 4-rank of the narrow ideal class group of a quadratic field, J. of Number Th. 31, 167-173(1989).

[13] E.Weiss, Algebraic Number Theory, McGraw-Hill Book Company.Inc, 1963.

[14] Q.Yue, Elements of order 4 of the Hilbert kernel in quadratic number fields, Acta Arith. 97 (2001), no.4, 295-311.

[15] -, The structure of certain $K_{2} O_{F}$, J. of Mathematical Research \& Exposition (Chinese), Vol.21,No.1 (2001), 1-6.

[16] -, Dyadic ideal, class group, tame kernel in quadratic number fields, J. Pure and Applied Algebra, Vol 166 No.1-2 (2002), 229-238.

[17] Q.Yue and K.Feng, The 4-rank of the tame kernel versus the 4-rank of the narrow class group in quadratic number fields, Acta Arith. 96(2000), no.2, 155-165. 